\title{
AN IMPROVED UPPER BOUND FOR THE ERROR IN THE ZERO-COUNTING FORMULAE FOR DIRICHLET $L$-FUNCTIONS AND DEDEKIND ZETA-FUNCTIONS
}

\author{
T. S. TRUDGIAN
}

Abstract. This paper contains new explicit upper bounds for the number of zeroes of Dirichlet $L$-functions and Dedekind zeta-functions in rectangles.

\section{INTRODUCTION AND RESULTS}

This paper pertains to the functions $N(T, \chi)$ and $N_{K}(T)$, respectively the number of zeroes $\rho=\beta+i \gamma$ of $L(s, \chi)$ and of $\zeta_{K}(s)$ in the region $0<\beta<1$ and $|\gamma| \leq T$. The purpose of this paper is to prove the following two theorems.

Theorem 1. Let $T \geq 1$ and $\chi$ be a primitive nonprincipal character modulo $k$. Then

$$
\left|N(T, \chi)-\frac{T}{\pi} \log \frac{k T}{2 \pi e}\right| \leq 0.315 \log k T+6.455 .
$$

In addition, if the right side of (1.1) is written as $C_{1} \log k T+C_{2}$, one may use the values of $C_{1}$ and $C_{2}$ contained in Table 1 .

Theorem 2. Let $T \geq 1$ and $K$ be a number field with degree $n_{K}=[K: \mathbb{Q}]$ and absolute discriminant $d_{K}$. Then

$\left|N_{K}(T)-\frac{T}{\pi} \log \left\{d_{K}\left(\frac{T}{2 \pi e}\right)^{n_{K}}\right\}\right| \leq 0.316\left\{\log d_{K}+n_{K} \log T\right\}+5.872 n_{K}+3.655$.

In addition, if the right side of (1.2) is written as $D_{1}\left\{\log d_{K}+n_{K} \log T\right\}+D_{2} n_{K}+$ $D_{3}$, one may use the values of $D_{1}, D_{2}$ and $D_{3}$ contained in Table 2 .

Theorem 1 and Table 1 improve on a result due to McCurley [3, Thm. 2.1]; Theorem 2 and Table 2 improve on a result due to Kadiri and Ng [2, Thm. 1]. The values of $C_{1}$ and $D_{1}$ given above are less than half of the corresponding values in [3] and [2. The improvement is due to Backlund's trick - explained in 43 - and some minor optimisation.

Explicit expressions for $C_{1}$ and $C_{2}$ and for $D_{1}, D_{2}$ and $D_{3}$ are contained in (4.11) and (4.12) and in (5.11) and (5.12). These contain a parameter $\eta$ which, when varied, gives rise to Tables 1 and 2 . The values in the right sides of (1.1) and (1.2) correspond to $\eta=\frac{1}{4}$ in the tables. Note that some minor improvement in the

Received by the editor June 8, 2012 and, in revised form, November 7, 2012, April 16, 2013, and August 12, 2013.

2010 Mathematics Subject Classification. Primary 11M06; Secondary 11M26, 11R42.

Key words and phrases. Zero-counting formula, Dirichlet $L$-functions, Dedekind zetafunctions.

Supported by Australian Research Council DECRA Grant DE120100173. 
lower order terms is possible if $T \geq T_{0}>1$; Tables 1 and 2 give this improvement when $T \geq 10$.

TABle 1. $C_{1}$ and $C_{2}$ in Theorem 1 and in 3 . for various values of $\eta$

\begin{tabular}{cccccc}
\hline \hline$\eta$ & \multicolumn{2}{c}{ McCurley $\left[\begin{array}{l}3] \\
C_{2}\end{array}\right.$} & \multicolumn{2}{c}{ When $T \geq 1$} & When $T \geq 10$ \\
& $C_{1}$ & $C_{1}$ & $C_{2}$ & $C_{2}$ \\
\hline 0.05 & 0.506 & 16.989 & 0.247 & 9.359 & 8.949 \\
0.10 & 0.552 & 13.202 & 0.264 & 8.049 & 7.640 \\
0.15 & 0.597 & 11.067 & 0.281 & 7.323 & 6.914 \\
0.20 & 0.643 & 9.606 & 0.298 & 6.828 & 6.419 \\
0.25 & 0.689 & 8.509 & 0.315 & 6.455 & 6.046 \\
0.30 & 0.735 & 7.641 & 0.332 & 6.156 & 5.747 \\
0.35 & 0.781 & 6.929 & 0.349 & 5.907 & 5.498 \\
0.40 & 0.827 & 6.330 & 0.365 & 5.694 & 5.284 \\
0.45 & 0.873 & 5.817 & 0.382 & 5.506 & 5.096 \\
0.50 & 0.919 & 5.370 & 0.399 & 5.338 & 4.928 \\
\hline \hline
\end{tabular}

TABLE 2. $D_{1}, D_{2}$ and $D_{3}$ in Theorem 2 and in [2] for various values of $\eta$

\begin{tabular}{ccccccccc}
\hline \hline$\eta$ & \multicolumn{3}{c}{ Kadiri and Ng [2] } & \multicolumn{4}{c}{ When $T \geq 1$} & \multicolumn{2}{c}{ When $T \geq 10$} \\
& $D_{1}$ & $D_{2}$ & $D_{3}$ & $D_{1}$ & $D_{2}$ & $D_{3}$ & $D_{2}$ & $D_{3}$ \\
\hline 0.05 & 0.506 & 16.95 & 7.663 & 0.247 & 8.851 & 3.024 & 8.726 & 2.081 \\
0.10 & 0.552 & 13.163 & 7.663 & 0.265 & 7.521 & 3.178 & 7.396 & 2.101 \\
0.15 & 0.597 & 11.029 & 7.663 & 0.282 & 6.776 & 3.335 & 6.651 & 2.123 \\
0.20 & 0.643 & 9.567 & 7.663 & 0.299 & 6.262 & 3.494 & 6.138 & 2.146 \\
0.25 & 0.689 & 8.471 & 7.663 & 0.316 & 5.872 & 3.655 & 5.748 & 2.171 \\
0.30 & 0.735 & 7.603 & 7.663 & 0.333 & 5.557 & 3.818 & 5.433 & 2.197 \\
0.35 & 0.781 & 6.891 & 7.663 & 0.350 & 5.292 & 3.983 & 5.169 & 2.224 \\
0.40 & 0.827 & 6.292 & 7.663 & 0.367 & 5.062 & 4.149 & 4.939 & 2.253 \\
0.45 & 0.873 & 5.778 & 7.663 & 0.384 & 4.859 & 4.317 & 4.737 & 2.283 \\
0.50 & 0.919 & 5.331 & 7.663 & 0.401 & 4.676 & 4.485 & 4.554 & 2.315 \\
\hline \hline
\end{tabular}

Explicit estimation of the error terms of the zero-counting function for $L(s, \chi)$ is done in $§ 2$. Backlund's trick is modified to suit Dirichlet $L$-functions in $\$ 3$. Theorem 1 is proved in $\$ 4$. Theorem 2 is proved in \$5.

The Riemann zeta-function, $\zeta(s)$, is both a Dirichlet $L$-function (albeit to the principal character) and a Dedekind zeta-function. The error term in the zero counting function for $\zeta(s)$ has been improved, most recently, by the author 7 . One can estimate the error term in the case of $\zeta(s)$ more efficiently owing to explicit bounds on $\zeta(1+i t)$, for $t \gg 1$. It would be of interest to see whether such bounds for $L(1+i t, \chi)$ and $\zeta_{K}(1+i t)$ could be produced relatively easily — this would lead to an improvement of the results in this paper. 


\section{Estimating $N(T, \chi)$}

Let $\chi$ be a primitive nonprincipal character modulo $k$, and let $L(s, \chi)$ be the Dirichlet $L$-series attached to $\chi$. Let $a=(1-\chi(-1)) / 2$ so that $a$ is 0 or 1 according to whether $\chi$ is an even or an odd character. Then the function

$$
\xi(s, \chi)=\left(\frac{k}{\pi}\right)^{(s+a) / 2} \Gamma\left(\frac{s+a}{2}\right) L(s, \chi)
$$

is entire and satisfies the functional equation

$$
\xi(1-s, \bar{\chi})=\frac{i^{a} k^{1 / 2}}{\tau(\chi)} \xi(s, \chi)
$$

where $\tau(\chi)=\sum_{n=1}^{k} \chi(n) \exp (2 \pi i n / k)$.

Let $N(T, \chi)$ denote the number of zeroes $\rho=\beta+i \gamma$ of $L(s, \chi)$ for which $0<\beta<1$ and $|\gamma| \leq T$. For any $\sigma_{1}>1$ form the rectangle $R$ having vertices at $\sigma_{1} \pm i T$ and $1-\sigma_{1} \pm i T$, and let $\mathcal{C}$ denote the portion of the boundary of the rectangle in the region $\sigma \geq \frac{1}{2}$. From Cauchy's theorem and (2.2) one deduces that

$$
N(T, \chi)=\frac{1}{\pi} \Delta_{\mathcal{C}} \arg \xi(s, \chi)
$$

Thus

$$
\begin{aligned}
N(T, \chi) & =\frac{1}{\pi}\left\{\Delta_{\mathcal{C}} \arg \left(\frac{k}{\pi}\right)^{(s+a) / 2}+\Delta_{\mathcal{C}} \arg \Gamma\left(\frac{s+a}{2}\right)+\Delta_{\mathcal{C}} \arg L(s, \chi)\right\} \\
& =\frac{T}{\pi} \log \frac{k}{\pi}+\frac{2}{\pi} \Im \log \Gamma\left(\frac{1}{4}+\frac{a}{2}+i \frac{T}{2}\right)+\frac{1}{\pi} \Delta_{\mathcal{C}} \arg L(s, \chi) .
\end{aligned}
$$

To evaluate the second term on the right side of (2.3) one needs an explicit version of Stirling's formula. Such a version is provided in [4, p. 294]; to wit,

$$
\log \Gamma(z)=\left(z-\frac{1}{2}\right) \log z-z+\frac{1}{2} \log 2 \pi+\frac{\theta}{6|z|},
$$

which is valid for $|\arg z| \leq \frac{\pi}{2}$, and in which $\theta$ denotes a complex number satisfying $|\theta| \leq 1$. Using (2.4) one obtains

$$
\begin{aligned}
\Im \log \Gamma\left(\frac{1}{4}+\frac{a}{2}+i \frac{T}{2}\right)= & \frac{T}{2} \log \frac{T}{2 e}+\frac{T}{4} \log \left(1+\frac{(2 a+1)^{2}}{4 T^{2}}\right) \\
& +\frac{2 a-1}{4} \tan ^{-1}\left(\frac{2 T}{2 a+1}\right)+\frac{\theta}{3\left|\frac{1}{2}+a+i T\right|} .
\end{aligned}
$$

Denote the last three terms in (2.5) by $g(a, T)$. Using elementary calculus one can show that $|g(0, T)| \leq g(1, T)$ and that $g(1, T)$ is decreasing for $T \geq 1$. This, together with (2.3) and (2.5), shows that

$$
\left|N(T, \chi)-\frac{T}{\pi} \log \frac{k T}{2 \pi e}\right| \leq \frac{1}{\pi}\left|\Delta_{\mathcal{C}} \arg L(s, \chi)\right|+\frac{2}{\pi} g(1, T) .
$$

All that remains is to estimate $\Delta_{\mathcal{C}} \arg L(s, \chi)$. Write $\mathcal{C}$ as the union of three straight lines; viz. let $\mathcal{C}=\mathcal{C}_{1} \cup \mathcal{C}_{2} \cup \mathcal{C}_{3}$, where $\mathcal{C}_{1}$ connects $\frac{1}{2}-i T$ to $\sigma_{1}-i T, \mathcal{C}_{2}$ connects $\sigma_{1}-i T$ to $\sigma_{1}+i T$, and $\mathcal{C}_{3}$ connects $\sigma_{1}+i T$ to $\frac{1}{2}+i T$. Since $L(\bar{s}, \chi)=\overline{L(s, \bar{\chi})}$ 
a bound for the integral on $\mathcal{C}_{3}$ will serve as a bound for that on $\mathcal{C}_{1}$. Estimating the contribution along $\mathcal{C}_{2}$ poses no difficulty since

$$
\left|\arg L\left(\sigma_{1}+i t, \chi\right)\right| \leq\left|\log L\left(\sigma_{1}+i t, \chi\right)\right| \leq \log \zeta\left(\sigma_{1}\right) .
$$

To estimate $\Delta_{\mathcal{C}_{3}} \arg L(s, \chi)$ define

$$
f(s)=\frac{1}{2}\left\{L(s+i T, \chi)^{N}+L(s-i T, \bar{\chi})^{N}\right\},
$$

for some positive integer $N$, to be determined later. Thus $f(\sigma)=\Re L(\sigma+i T, \chi)^{N}$. Suppose that there are $n$ zeroes of $\Re L(\sigma+i T, \chi)^{N}$ for $\sigma \in\left[\frac{1}{2}, \sigma_{1}\right]$. These zeroes partition the segment into $n+1$ intervals. On each interval $\arg L(\sigma+i T, \chi)^{N}$ can increase by at most $\pi$. Thus

$$
\left|\Delta_{\mathcal{C}_{3}} \arg L(s, \chi)\right|=\frac{1}{N}\left|\Delta_{\mathcal{C}_{3}} \arg L(s, \chi)^{N}\right| \leq \frac{(n+1) \pi}{N},
$$

whence (2.6) may be written as

$$
\left|N(T, \chi)-\frac{T}{\pi} \log \frac{k T}{2 \pi e}\right| \leq \frac{2}{\pi}\left\{\log \zeta\left(\sigma_{1}\right)+g(1, T)\right\}+\frac{2(n+1)}{N} .
$$

One may estimate $n$ with Jensen's formula.

Lemma 1 (Jensen's formula). Let $f(z)$ be holomorphic for $|z-a| \leq R$ and nonvanishing at $z=a$. Let the zeroes of $f(z)$ inside the circle be $z_{k}$, where $1 \leq k \leq n$, and let $\left|z_{k}-a\right|=r_{k}$. Then

$$
\log \frac{R^{n}}{\left|r_{1} r_{2} \cdots r_{n}\right|}=\frac{1}{2 \pi} \int_{0}^{2 \pi} \log f\left(a+R e^{i \phi}\right) d \phi-\log |f(a)| .
$$

This is done in 4

\section{BACKLUND'S TRICK}

For a complex-valued function $F(s)$ and for $\delta>0$, define $\Delta_{+} \arg F(s)$ to be the change in argument of $F(s)$ as $\sigma$ varies from $\frac{1}{2}$ to $\frac{1}{2}+\delta$, and define $\Delta_{-} \arg F(s)$ to be the change in argument of $F(s)$ as $\sigma$ varies from $\frac{1}{2}$ to $\frac{1}{2}-\delta$.

Backlund's trick is to show that if there are zeroes of $\Re F(\sigma+i T)^{N}$ on the line $\sigma \in\left[\frac{1}{2}, \sigma_{1}\right]$, then there are zeroes on the line $\sigma \in\left[1-\sigma_{1}, \frac{1}{2}\right]$. This device was introduced by Backlund in [1] for the Riemann zeta-function.

Following Backlund's approach one can prove the following general lemma.

Lemma 2. Let $N$ be a positive integer and let $T \geq T_{0} \geq 1$. Suppose that there is an upper bound $E$ that satisfies

$$
\left|\Delta_{+} \arg F(s)+\Delta_{-} \arg F(s)\right| \leq E,
$$

where $E=E\left(\delta, T_{0}\right)$. Suppose further that there exists an $n \geq 3+\lfloor N E / \pi\rfloor$ for which

$$
n \pi \leq\left|\Delta_{\mathcal{C}_{3}} \arg F(s)^{N}\right|<(n+1) \pi .
$$

Then there are at least $n$ distinct zeroes of $\Re F(\sigma+i T)^{N}$, denoted by $\rho_{\nu}=a_{\nu}+$ $i T$ (where $1 \leq \nu \leq n$ and $\frac{1}{2} \leq a_{n}<a_{n-1}<\cdots \leq \sigma_{1}$ ), such that the bound $\left|\Delta \arg F(s)^{N}\right| \geq \nu \pi$ is achieved for the first time when $\sigma$ passes over $a_{\nu}$ from above.

In addition there are at least $n-2-\lfloor N E / \pi\rfloor$ distinct zeroes $\rho_{\nu}^{\prime}=a_{\nu}^{\prime}+i T$ (where $1 \leq \nu \leq n-2$ and $\left.1-\sigma_{1} \leq a_{1}^{\prime}<a_{2}^{\prime}<\cdots \leq \frac{1}{2}\right)$. 
Moreover,

$$
a_{\nu} \geq 1-a_{\nu}^{\prime}, \quad \text { for } \nu=1,2, \ldots, n-2-\lfloor N E / \pi\rfloor,
$$

and, if $\eta$ is defined by $\sigma_{1}=\frac{1}{2}+\sqrt{2}\left(\eta+\frac{1}{2}\right)$, then

$$
\prod_{\nu=1}^{n}\left|1+\eta-a_{\nu}\right| \prod_{\nu=1}^{n-2-\lfloor N E / \pi\rfloor}\left|1+\eta-a_{\nu}^{\prime}\right| \leq\left(\frac{1}{2}+\eta\right)^{2 n-2-\lfloor N E / \pi\rfloor} .
$$

Proof. It follows from (3.1) that $\left|\arg F(s)^{N}\right|$ must increase as $\sigma$ varies from $\sigma_{1}$ to $\frac{1}{2}$. This increase may only occur if $\sigma$ has passed over a zero of $\Re F(s)^{N}$, irrespective of its multiplicity. In particular, as $\sigma$ moves along $\mathcal{C}_{3}$,

$$
\left|\Delta \arg F(s)^{N}\right| \geq \pi, 2 \pi, \ldots, n \pi .
$$

Let $\rho_{\nu}=a_{\nu}+i t$ denote the distinct zeroes of $\Re F(s)^{N}$, the passing over of which produces, for the first time, the bound $\left|\Delta \arg F(s)^{N}\right| \geq \nu \pi$. It follows that there must be $n$ such points, and that $\frac{1}{2} \leq a_{n}<a_{n-1}<\ldots<a_{2}<a_{1} \leq \sigma_{1}$. Also if $\frac{1}{2}+\delta \geq a_{\nu}$, then

$$
\left|\Delta_{+} \arg F(s)^{N}\right| \geq(n-\nu) \pi .
$$

Equation (3.4) is true when $\nu=n$ and so, by the definition of $\rho_{\nu}$, it is true for all $1 \leq \nu \leq n$.

By the hypothesis in Lemma 2

$$
\left|\Delta_{+} \arg F(s)^{N}+\Delta_{-} \arg F(s)^{N}\right| \leq N E .
$$

When $\frac{1}{2}+\delta \geq a_{\nu}$, (3.4) and (3.5) show that

$$
\left|\Delta_{-} \arg F(s)^{N}\right| \geq(n-\nu-N E / \pi) \pi,
$$

for $1 \leq \nu \leq n-2-\lfloor N E / \pi\rfloor$. When $\frac{1}{2}+\delta=a_{\nu}$ and $\nu=n-2-\lfloor N E / \pi\rfloor$, it follows from (3.6) that $\left|\Delta_{-} \arg F(s)^{N}\right| \geq \pi$. The increase in the argument is only possible if there is a zero of $\Re F(s)^{N}$, the real part of which is greater than $\frac{1}{2}-\delta=1-a_{n-2-\lfloor N E / \pi\rfloor}$. Label this zero $\rho_{n-2-\lfloor N E / \pi\rfloor}^{\prime}=a_{n-2-\lfloor N E / \pi\rfloor}^{\prime}+i T$. Repeat the procedure when $\nu=n-3-\lfloor N E / \pi\rfloor, \ldots, 2,1$, whence (3.2) follows. This produces a positive number of zeroes in $\left[1-\sigma_{1}, \frac{1}{2}\right]$ provided that $n \geq 3+\lfloor N E / \pi\rfloor$.

For zeroes $\rho_{\nu}$ lying to the left of $1+\eta$ one has

$$
\left|1+\eta-a_{\nu}\right|\left|1+\eta-a_{\nu}^{\prime}\right| \leq\left(1+\eta-a_{\nu}\right)\left(\eta+a_{\nu}\right),
$$

by (3.2). This is a decreasing function for $a_{\nu} \in\left[\frac{1}{2}, 1+\eta\right]$ and so, for these zeroes,

$$
\left|1+\eta-a_{\nu}\right|\left|1+\eta-a_{\nu}^{\prime}\right| \leq\left(\frac{1}{2}+\eta\right)^{2} \text {. }
$$

For zeroes lying to the right of $1+\eta$ one has

$$
\left|1+\eta-a_{\nu}\right|\left|1+\eta-a_{\nu}^{\prime}\right| \leq\left(a_{\nu}-1-\eta\right)\left(\eta+a_{\nu}\right) .
$$

This is increasing with $a_{\nu}$ and so, for these zeroes,

$$
\left|1+\eta-a_{\nu}\right|\left|1+\eta-a_{\nu}^{\prime}\right| \leq \sigma_{1}^{2}-\sigma_{1}-\eta(1+\eta) .
$$

The bounds in (3.7) and (3.8) are equal 1 when $\sigma_{1}=\frac{1}{2}+\sqrt{2}\left(\eta+\frac{1}{2}\right)$. Thus (3.3) holds for $\sigma_{1}=\frac{1}{2}+\sqrt{2}\left(\eta+\frac{1}{2}\right)$. For the unpaired zeroes one may use the bound $\left|1+\eta-a_{\nu}\right| \leq \frac{1}{2}+\eta$, whence (3.3) follows.

\footnotetext{
${ }^{1}$ McCurley does not use Backlund's trick. Accordingly, his upper bounds in place of (3.7) and (3.8) are $\frac{1}{2}+\eta$ and $\sigma_{1}-1-\eta$. These are equal at $\sigma_{1}=\frac{3}{2}+2 \eta$, which is his choice of $\sigma_{1}$.
} 
3.1. Applying Backlund's trick. Apply Jensen's formula on the function $F(s)$, with $a=1+\eta$ and $R=r\left(\frac{1}{2}+\eta\right)$, where $r>1$. Assume that the hypotheses of Lemma 2 hold. If $1+\eta-r\left(\frac{1}{2}+\eta\right) \leq 1-\sigma_{1}$, then all of the $2 n-1-\lfloor N E / \pi\rfloor$ zeroes of $\Re F(\sigma+i T)^{N}$ are included in the contour. Thus the left side of (2.9) is

$$
\begin{aligned}
& \log \frac{\left\{r\left(\frac{1}{2}+\eta\right)\right\}^{2 n-2-\lfloor N E / \pi\rfloor}}{\left|1+\eta-a_{1}\right| \cdots\left|1+\eta-a_{n}\right|\left|1+\eta-a_{1}^{\prime}\right| \cdots\left|1+\eta-a_{n-2-\lfloor N E / \pi\rfloor}^{\prime}\right|} \\
& \geq(2 n-2-\lfloor N E / \pi\rfloor) \log r,
\end{aligned}
$$

by (3.3). If the contour does not enclose all of the $2 n-2-[N E / \pi]$ zeroes of $\Re F(\sigma+i T)^{N}$, then the following argument, thoughtfully provided by Professor D.R. Heath-Brown, allows one still to make a saving.

To a zero at $x+i t$, with $\frac{1}{2} \leq x \leq 1+\eta$ one may associate a zero at $x^{\prime}+i t$ where, by (3.2), $1-x \leq x^{\prime} \leq \frac{1}{2}$. Thus, for an intermediate radius, zeroes to the right of $\frac{1}{2}$ yet still close to $\frac{1}{2}$ will have their pairs included in the contour. Let $X$ satisfy $1+\eta-\left(\frac{1}{2}+\eta\right) / r<X<\min \left\{1+\eta, r\left(\frac{1}{2}+\eta\right)-\eta\right\}$. Since $r>1$, this guarantees that $X>\frac{1}{2}$. For a zero at $x+i t$ consider two cases: $x \geq X$ and $x<X$.

In the former, there is no guarantee that the paired zero $x^{\prime}+i t$ is included in the contour. Thus the zero at $x+i t$ is counted in Jensen's formula with weight

$$
\log \frac{r\left(\frac{1}{2}+\eta\right)}{1+\eta-x} \geq \log \frac{r\left(\frac{1}{2}+\eta\right)}{1+\eta-X} .
$$

Now, when $x<X$, the paired zero at $x^{\prime}$ is included in the contour, since $1+\eta-$ $r\left(\frac{1}{2}+\eta\right)<1-X<1-x \leq x^{\prime}$. Thus, in Jensen's formula, the contribution is

$$
\begin{aligned}
\log \frac{r\left(\frac{1}{2}+\eta\right)}{1+\eta-x}+\log \frac{r\left(\frac{1}{2}+\eta\right)}{1+\eta-x^{\prime}} & \geq \log \frac{r\left(\frac{1}{2}+\eta\right)}{1+\eta-x}+\log \frac{r\left(\frac{1}{2}+\eta\right)}{\eta+x} \\
& =\log \frac{r^{2}\left(\frac{1}{2}+\eta\right)^{2}}{(1+\eta-x)(\eta+x)} .
\end{aligned}
$$

The function appearing in the denominator of (3.11) is decreasing for $x \geq \frac{1}{2}$. Thus the zeroes at $x+i t$ and $x^{\prime}+i t$ contribute at least $2 \log r$.

Suppose now that there are $n$ zeroes in $\left[\frac{1}{2}, \sigma_{1}\right]$, and that there are $k$ zeroes, the real parts of which are at least $X$. The contribution of all the zeroes ensnared by the integral in Jensen's formula is at least

$$
k \log \frac{r\left(\frac{1}{2}+\eta\right)}{1+\eta-X}+2(n-k) \log r=k \log \frac{\left(\frac{1}{2}+\eta\right)}{r(1+\eta-X)}+2 n \log r \geq 2 n \log r,
$$

which implies (3.9)

3.2. Calculation of $E$ in Lemma 2, From (2.1) and (2.2) it follows that

$$
\Delta_{+} \arg \xi(s, \chi)=-\Delta_{-} \arg \xi(s, \chi) .
$$

Since $\arg (\pi / k)^{-\frac{s+a}{2}}=-\frac{t}{2} \log (\pi / k)$ then $\Delta_{ \pm}(\pi / k)^{-\frac{s+a}{2}}=0$, whence

$$
\left|\Delta_{+} \arg L(s, \chi)+\Delta_{-} \arg L(s, \chi)\right|=\left|\Delta_{+} \arg \Gamma\left(\frac{s+a}{2}\right)+\Delta_{-} \arg \Gamma\left(\frac{s+a}{2}\right)\right| .
$$

Using (2.4) one may write

$$
\left|\Delta_{+} \arg \Gamma\left(\frac{s+a}{2}\right)+\Delta_{-} \arg \Gamma\left(\frac{s+a}{2}\right)\right| \leq G(a, \delta, t),
$$


where

$$
\begin{aligned}
G(a, \delta, t)= & \frac{1}{2}\left(a-\frac{1}{2}+\delta\right) \tan ^{-1} \frac{a+\frac{1}{2}+\delta}{t}+\frac{1}{2}\left(a-\frac{1}{2}-\delta\right) \tan ^{-1} \frac{a+\frac{1}{2}-\delta}{t} \\
& -\left(a-\frac{1}{2}\right) \tan ^{-1} \frac{a+\frac{1}{2}}{t}-\frac{t}{4} \log \left[1+\frac{2 \delta^{2}\left\{t^{2}-\left(\frac{1}{2}+a\right)^{2}\right\}+\delta^{4}}{\left\{t^{2}+\left(\frac{1}{2}+a\right)^{2}\right\}^{2}}\right] \\
& +\frac{1}{3}\left\{\frac{1}{\left|\frac{1}{2}+\delta+a+i t\right|}+\frac{1}{\left|\frac{1}{2}-\delta+a+i t\right|}+\frac{2}{\left|\frac{1}{2}+a+i t\right|}\right\} .
\end{aligned}
$$

One can show that $G(a, \delta, t)$ is decreasing in $t$ and increasing in $\delta$, and that $G(1, \delta, t) \leq G(0, \delta, t)$. Therefore, since, in Lemma 2, one takes $\sigma_{1}=\frac{1}{2}+\sqrt{2}\left(\frac{1}{2}+\eta\right)$, it follows that $\delta=\sqrt{2}\left(\frac{1}{2}+\eta\right)$, whence one may take

$$
E=G\left(0, \sqrt{2}\left(\frac{1}{2}+\eta\right), t_{0}\right),
$$

for $t \geq t_{0}$.

\section{Proof of Theorem 1}

First, suppose that $\left|\Delta_{\mathcal{C}_{3}} \arg L(s, \chi)^{N}\right|<3+\lfloor N E / \pi\rfloor$. Thus (2.6) becomes

$$
\left|N(T, \chi)-\frac{T}{\pi} \log \frac{k T}{2 \pi e}\right| \leq \frac{2}{\pi}\left\{\log \zeta\left(\sigma_{1}\right)+g(1, T)+E\right\}+\frac{6}{N} .
$$

Now suppose that $\left|\Delta_{\mathcal{C}_{3}} \arg L(s, \chi)^{N}\right| \geq 3+\lfloor N E / \pi\rfloor$, whence Lemma 2 may be applied.

To apply Jensen's formula to the function $f(s)$, defined in (2.7), it is necessary to show that $f(1+\eta)$ is nonzero; this is easy to do upon invoking an observation due to Rosser [6]. Write $L(1+\eta+i T, \chi)=K e^{i \psi}$, where $K>0$. Choose a sequence of $N$ 's tending to infinity for which $N \psi$ tends to zero modulo $2 \pi$. Thus

$$
\frac{f(1+\eta)}{|L(1+\eta+i T, \chi)|^{N}} \rightarrow 1
$$

Since $\chi$ is a primitive nonprincipal character then $f(s)$ is holomorphic on the circle. It follows from (2.9) and (3.9) that

$$
n \leq \frac{1}{4 \pi \log r} J-\frac{1}{2 \log r} \log |f(1+\eta)|+1+\frac{N E}{2 \pi},
$$

where

$$
J=\int_{-\frac{\pi}{2}}^{\frac{3 \pi}{2}} \log \left|f\left(1+\eta+r\left(\frac{1}{2}+\eta\right) e^{i \phi}\right)\right| d \phi .
$$

Write $J=J_{1}+J_{2}$, where the respective ranges of integration of $J_{1}$ and $J_{2}$ are $\phi \in[-\pi / 2, \pi / 2]$ and $\phi \in[\pi / 2,3 \pi / 2]$. For $\sigma>1$

$$
\frac{\zeta(2 \sigma)}{\zeta(\sigma)} \leq|L(s, \chi)| \leq \zeta(\sigma)
$$

which shows that

$$
J_{1} \leq N \int_{-\pi / 2}^{\pi / 2} \log \zeta\left(1+\eta+r\left(\frac{1}{2}+\eta\right) \cos \phi\right) d \phi .
$$

On $J_{2}$ use

$$
\log |f(s)| \leq N \log |L(s+i T, \chi)|
$$


and the convexity bound [5, Thm. 3]

$$
|L(s, \chi)| \leq\left(\frac{k|s+1|}{2 \pi}\right)^{(1+\eta-\sigma) / 2} \zeta(1+\eta),
$$

valid for $-\eta \leq \sigma \leq 1+\eta$, where $0<\eta \leq \frac{1}{2}$, to show that

$$
J_{2} \leq \pi N \log \zeta(1+\eta)+N \frac{r\left(\frac{1}{2}+\eta\right)}{2} \int_{\pi / 2}^{3 \pi / 2}(-\cos \phi) \log \left\{\frac{k T w(T, \phi, \eta, r)}{2 \pi}\right\} d \phi
$$

where

$$
\begin{aligned}
& w(T, \phi, \eta, r)^{2} \\
& \quad=1+\frac{2 r\left(\frac{1}{2}+\eta\right) \sin \theta}{T}+\frac{r^{2}\left(\frac{1}{2}+\eta\right)^{2}+(2+\eta)^{2}+2 r\left(\frac{1}{2}+\eta\right)(2+\eta) \cos \theta}{T^{2}} .
\end{aligned}
$$

For $\phi \in[\pi / 2, \pi]$, the function $w(T, \phi, \eta, r)$ is decreasing in $T$; for $\phi \in[\pi, 3 \pi / 2]$ it is bounded above by $w^{*}(T, \phi, \eta, r)$, where

$$
w^{*}(T, \phi, \eta, r)^{2}=1+\frac{r^{2}\left(\frac{1}{2}+\eta\right)^{2}+(2+\eta)^{2}+2 r\left(\frac{1}{2}+\eta\right)(2+\eta) \cos \theta}{T^{2}},
$$

which is decreasing in $T$.

To bound $n$ using (4.3) it remains to bound $-\log |f(1+\eta)|$. This is done by using (4.2) and (4.4) to show that

$$
-\log |f(1+\eta)| \rightarrow-N \log |L(1+\eta+i T)| \leq-N \log [\zeta(2+2 \eta) / \zeta(1+\eta)] .
$$

This, together with (2.8), (4.1), (4.3), (4.5), (4.7) and sending $N \rightarrow \infty$, shows that, when $T \geq T_{0}$,

$$
\left|N(T, \chi)-\frac{T}{\pi} \log \frac{k T}{2 \pi e}\right| \leq \frac{r\left(\frac{1}{2}+\eta\right)}{2 \pi \log r} \log k T+C_{2},
$$

where

$$
\begin{aligned}
C_{2}=\frac{2}{\pi} & \left\{\log \zeta\left(\frac{1}{2}+\sqrt{2}\left(\frac{1}{2}+\eta\right)\right)+g(1, T)+\frac{E}{2}\right\}+\frac{3}{2 \log r} \log \zeta(1+\eta) \\
& -\frac{\log \zeta(2+2 \eta)}{\log r}+\frac{1}{2 \pi \log r} \int_{-\pi / 2}^{\pi / 2} \log \zeta\left(1+\eta+r\left(\frac{1}{2}+\eta\right) \cos \phi\right) d \phi \\
& +\frac{r\left(\frac{1}{2}+\eta\right)}{4 \pi \log r}\left\{-2 \log 2 \pi+\int_{\pi / 2}^{\pi}(-\cos \phi) \log w\left(T_{0}, \phi, \eta, r\right) d \phi\right. \\
& \left.+\int_{\pi}^{3 \pi / 2}(-\cos \phi) \log w^{*}\left(T_{0}, \phi, \eta, r\right) d \phi\right\} .
\end{aligned}
$$

4.1. A small improvement. Consider that what is really sought is a number $p$ satisfying $-\eta \leq p<0$ for which one can bound $L(p+i t, \chi)$, provided that $1+\eta-r\left(\frac{1}{2}+\eta\right) \geq p$. Indeed the restriction that $p \geq-\eta$ can be relaxed by adapting the convexity bound, but, as will be shown soon, this is unnecessary.

The convexity bound (4.6) becomes the rather ungainly

$$
|L(s, \chi)| \leq\left\{\left(\frac{k|1+s|}{2 \pi}\right)^{(1 / 2-p)(1+\eta-\sigma)} \zeta(1-p)^{1+\eta-\sigma} \zeta(1+\eta)^{\sigma-p}\right\}^{1 /(1+\eta-p)}
$$


valid for $-\eta \leq p \leq \sigma \leq 1+\eta$. Such an alternation only changes $J_{2}$, whence the coefficient of $\log k T$ in (4.10) becomes

$$
\frac{r\left(\frac{1}{2}+\eta\right)\left(\frac{1}{2}-p\right)}{\pi(1+\eta-p) \log r} .
$$

This is minimised when $r=(1+\eta-p) /(1 / 2+\eta)$, whence (4.10) becomes

$$
\left|N(T, \chi)-\frac{T}{\pi} \log \frac{k T}{2 \pi e}\right| \leq \frac{\frac{1}{2}-p}{\pi \log \left(\frac{1+\eta-p}{1 / 2+\eta}\right)} \log k T+C_{2},
$$

where

$$
\begin{aligned}
& C_{2}=\frac{2}{\pi}\left\{\log \zeta\left(\frac{1}{2}+\sqrt{2}\left(\frac{1}{2}+\eta\right)\right)+g(1, T)+\frac{G\left(0, \sqrt{2}\left(\frac{1}{2}+\eta\right), T_{0}\right)}{2}\right\} \\
& +\frac{1}{\log \left(\frac{1+\eta-p}{1 / 2+\eta}\right)}\left\{\frac{3}{2} \log \zeta(1+\eta)-\log \zeta(2+2 \eta)+\frac{1}{\pi} \log \frac{\zeta(1-p)}{\zeta(1+\eta)}\right. \\
& +\frac{1}{2 \pi} \int_{-\pi / 2}^{\pi / 2} \log \zeta(1+\eta+(1+\eta-p) \cos \phi) d \phi+\frac{\frac{1}{2}-p}{2 \pi}(-2 \log 2 \pi \\
& \left.\left.+\int_{\pi / 2}^{\pi}(-\cos \phi) \log w\left(T_{0}, \phi, \eta, r\right) d \phi+\int_{\pi}^{3 \pi / 2}(-\cos \phi) \log w^{*}\left(T_{0}, \phi, \eta, r\right) d \phi\right)\right\},
\end{aligned}
$$

in which $g(1, T), G\left(a, \delta, T_{0}\right), w$ and $w^{*}$ are defined in (2.5), (3.13), (4.8) and (4.9).

The coefficient of $\log k T$ in (4.11) is minimal when $p=0$ and $r=\frac{1+\eta}{1 / 2+\eta}$. One cannot choose $p=0$ nor should one choose $p$ to be too small of a negative number lest the term $\log \zeta(1-p) / \zeta(1+\eta)$ become too large. Choosing $p=-\eta / 12$ ensures that $C_{2}$ in (4.11) is always smaller than the corresponding term in McCurley's proof. Theorem 1 follows upon taking $T_{0}=1$ and $T_{0}=10$. One could prove different bounds were one interested in 'large' values of $k T$. In this instance the term $C_{2}$ is not so important, whence one could choose a smaller value of $p$.

\section{The Dedekind ZeTA-FunCtion}

This section employs the notation of $\S \$ 2$. Consider a number field $K$ with degree $n_{K}=[K: \mathbb{Q}]$ and absolute discriminant $d_{K}$. In addition let $r_{1}$ and $r_{2}$ be the number of real and complex embeddings in $K$, whence $n_{K}=r_{1}+2 r_{2}$. Define the Dedekind zeta-function to be

$$
\zeta_{K}(s)=\sum_{\mathfrak{a} \subset \mathcal{O}_{K}} \frac{1}{(\mathbb{N a})^{s}},
$$

where $\mathfrak{a}$ runs over the nonzero ideals. The completed zeta-function

$$
\xi_{K}(s)=s(s-1)\left(\frac{d_{K}}{\pi^{n_{K}} 2^{2 r_{2}}}\right)^{s / 2} \Gamma(s / 2)^{r_{1}} \Gamma(s)^{r_{2}} \zeta_{K}(s)
$$

satisfies the functional equation

$$
\xi_{K}(s)=\xi_{K}(1-s) .
$$


Let $a(s)=(s-1) \zeta_{K}(s)$ and let

$$
f(\sigma)=\frac{1}{2}\left\{a(s+i T)^{N}+a(s-i T)^{N}\right\} .
$$

It follows from (5.1) and (5.2) that

$$
\left|\Delta_{+} \arg a(s)+\Delta_{-} \arg a(s)\right| \leq F(\delta, t)+n_{K} G(0, \delta, t),
$$

where $F(\delta, t)=2 \tan ^{-1} \frac{1}{2 t}-\tan ^{-1} \frac{1 / 2+\delta}{t}-\tan ^{-1} \frac{1 / 2-\delta}{t}$, and $G(0, \delta, t)$ is defined in (3.13).

Thus, following the arguments in $\S ₫ 2$, one arrives at

$$
\left|N_{K}(T)-\frac{T}{\pi} \log \left\{d_{K}\left(\frac{T}{2 \pi e}\right)^{n_{K}}\right\}\right| \leq \frac{2(n+1)}{N}+\frac{2 n_{K}}{\pi}\left\{|g(0, T)|+\log \zeta\left(\sigma_{1}\right)\right\}+2,
$$

where $n$ is bounded above by (4.3), in which $f(s)$ is defined in (5.3). Using the right inequality in

$$
\frac{\zeta_{K}(2 \sigma)}{\zeta_{K}(\sigma)} \leq\left|\zeta_{K}(s)\right| \leq\{\zeta(\sigma)\}^{n_{K}}
$$

one can show that the corresponding estimate for $J_{1}$ is

$$
J_{1} / N \leq \pi \log T+\int_{-\pi / 2}^{\pi / 2}\left\{\log \tilde{w}(T, \phi, \eta, r)+n_{K} \log \zeta\left(1+\eta+r\left(\frac{1}{2}+\eta\right) \cos \phi\right)\right\} d \phi,
$$

where

$$
\tilde{w}(T, \phi, \eta, r)^{2}=1+\frac{2 r\left(\frac{1}{2}+\eta\right) \sin \theta}{T}+\frac{r^{2}\left(\frac{1}{2}+\eta\right)^{2}+\eta^{2}+2 r \eta\left(\frac{1}{2}+\eta\right) \cos \theta}{T^{2}} .
$$

For $\phi \in[0, \pi / 2]$, the function $\tilde{w}(T, \phi, \eta, r)$ is decreasing in $T$; for $\phi \in[-\pi / 2,0]$ it is bounded above by $\tilde{w}^{*}(T, \phi, \eta, r)$, where

$$
\tilde{w}^{*}(T, \phi, \eta, r)^{2}=1+\frac{r^{2}\left(\frac{1}{2}+\eta\right)^{2}+\eta^{2}+2 r \eta\left(\frac{1}{2}+\eta\right) \cos \theta}{T^{2}}
$$

which is decreasing in $T$.

The integral $J_{2}$ is estimated using the following convexity result.

Lemma 3. Let $-\eta \leq p<0$. For $p \leq 1+\eta-r\left(\frac{1}{2}+\eta\right)$ the following bound holds:

$$
\begin{gathered}
|a(s)|^{1+\eta-p} \leq\left(\frac{1-p}{1+p}\right)^{1+\eta-\sigma} \zeta_{K}(1+\eta)^{\sigma-p} \zeta_{K}(1-p)^{1+\eta-\sigma}|1+s|^{1+\eta-p} \\
\times\left\{d\left(\frac{|1+s|}{2 \pi}\right)^{n_{K}}\right\}^{(1+\eta-\sigma)(1 / 2-p)}
\end{gathered}
$$

Proof. See [5, §7]. When $p=-\eta$ the bound reduces to that in [5, Thm. 4]. 
Using this it is straightforward to show that

$$
\begin{aligned}
J_{2} / N & \leq \frac{2 r\left(\frac{1}{2}+\eta\right)}{1+\eta-p}\left\{\log \frac{\zeta_{K}(1-p)}{\zeta_{k}(1+\eta)}+\log \frac{1-p}{1+p}+(1 / 2-p) \log \frac{d}{(2 \pi)^{n_{K}}}\right\} \\
& +\pi \zeta_{K}(1+\eta)+\log T\left(\pi+\frac{2 r n_{K}\left(\frac{1}{2}+\eta\right)\left(\frac{1}{2}-p\right)}{1+\eta-p}\right) \\
& +\int_{\pi / 2}^{3 \pi / 2} \log w\left(T_{0}, r, \eta, \phi\right) d \phi\left(1+\frac{n_{K} r\left(\frac{1}{2}+\eta\right)\left(\frac{1}{2}-p\right)(-\cos \phi)}{1+\eta-p}\right) d \phi .
\end{aligned}
$$

The quotient of Dedekind zeta-functions can be dispatched easily enough using

$$
-\frac{\zeta_{K}^{\prime}}{\zeta_{K}}(\sigma) \leq n_{K}\left\{-\frac{\zeta^{\prime}}{\zeta}(\sigma)\right\}
$$

to show that

$$
\log \frac{\zeta_{K}(1-p)}{\zeta_{K}(1+\eta)}=\int_{1-p}^{1+\eta}-\frac{\zeta_{K}^{\prime}}{\zeta_{K}}(\sigma) d \sigma \leq n_{K} \int_{1-p}^{1+\eta}-\frac{\zeta^{\prime}}{\zeta}(\sigma) d \sigma \leq n_{K} \log \frac{\zeta(1-p)}{\zeta(1+\eta)} .
$$

Finally the term $-\log |f(1+\eta)|$ is estimated as in the Dirichlet $L$-function case; cf. (4.2). This shows that

$$
\log |f(1+\eta)| \geq N \log \frac{\zeta_{K}(2+2 \eta)}{\zeta_{K}(1+\eta)}+\frac{N}{2} \log \left(\eta^{2}+T^{2}\right)+o(1) .
$$

This, together with (5.5), (5.7), (5.8), (5.9) and (5.10) and sending $N \rightarrow \infty$, shows that, when $T \geq T_{0}$,

$$
\begin{aligned}
\left|N_{K}(T)-\frac{T}{\pi} \log \left\{d_{K}\left(\frac{T}{2 \pi e}\right)^{n_{K}}\right\}\right| & \leq \frac{r\left(\frac{1}{2}+\eta\right)\left(\frac{1}{2}-p\right)}{\pi \log r(1+\eta-p)}\left\{\log d_{K}+n_{K} \log T\right\} \\
& +\left(C_{2}-\frac{2}{\pi}[g(1, T)-|g(0, T)|]\right) n_{K}+D_{3},
\end{aligned}
$$

where $C_{2}$ is given in (4.12) and

$$
\begin{aligned}
D_{3}=2+\frac{r\left(\frac{1}{2}+\eta\right)}{\pi \log r(1+\eta-p)} \log \left(\frac{1-p}{1+p}\right)+\frac{1}{\pi} F\left(\sqrt{2}\left(\frac{1}{2}+\eta\right), T_{0}\right) \\
+\frac{1}{2 \pi \log r}\left(\int_{-\pi / 2}^{0} \log \tilde{w}^{*}\left(T_{0}, r, \eta, \phi\right) d \phi+\int_{0}^{\pi / 2} \log \tilde{w}\left(T_{0}, r, \eta, \phi\right) d \phi\right. \\
\left.\quad+\int_{\pi / 2}^{\pi} \log w\left(T_{0}, r, \eta, \phi\right) d \phi+\int_{\pi}^{3 \pi / 2} \log w^{*}\left(T_{0}, r, \eta, \phi\right) d \phi\right) .
\end{aligned}
$$

If one chooses $p=-\eta / 9$, to ensure that the lower order terms in (5.11) are smaller than those in [2], one arrives at Theorem 2. One may choose a smaller value of $p$ if one is less concerned about the term $D_{2}$.

\section{ACKNOWLEDGEMEnTS}

The author would like to thank Professor Heath-Brown and Professors Ng and Kadiri for their advice. The author also thanks the referee for some constructive remarks. 


\section{REFERENCES}

[1] R. J. Backlund, Über die Nullstellen der Riemannschen Zetafunction, Acta Mathematica 41 (1916), no. 1, 345-375. MR 1555156

[2] Habiba Kadiri and Nathan Ng, Explicit zero density theorems for Dedekind zeta functions, J. Number Theory 132 (2012), no. 4, 748-775, DOI 10.1016/j.jnt.2011.09.002. MR.2887617

[3] Kevin S. McCurley, Explicit estimates for the error term in the prime number theorem for arithmetic progressions, Math. Comp. 42 (1984), no. 165, 265-285, DOI 10.2307/2007579. MR726004 (85e:11065)

[4] F. W. J. Olver, Asymptotics and Special Functions, Computer Science and Applied Mathematics. Academic Press [A subsidiary of Harcourt Brace Jovanovich, Publishers], New YorkLondon, 1974. MR0435697 (55 \#8655)

[5] Hans Rademacher, On the Phragmén-Lindelöf theorem and some applications, Math. Z 72 (1959/1960), 192-204. MR.0117200 (22 \#7982)

[6] Barkley Rosser, Explicit bounds for some functions of prime numbers, Amer. J. Math. 63 (1941), 211-232. MR0003018 (2,150e)

[7] Timothy Trudgian, An improved upper bound for the argument of the Riemann zeta-function on the critical line, Math. Comp. 81 (2012), no. 278, 1053-1061, DOI 10.1090/S0025-57182011-02537-8. MR2869049

Mathematical Sciences Institute, The Australian National University, Canberra, Australia, 0200

E-mail address: timothy.trudgian@anu.edu.au 